\title{
Un análisis económico de la intermediación en el sector turístico
}

\author{
Aleix Calveras \\ Departamento de Economía de la Empresa \\ Universitat de les Illes Balears \\ aleix.calveras@uib.es \\ Francina Orfila \\ Departamento de Economía de la Empresa \\ Universitat de les Illes Balears \\ francina.orfila@uib.es
}

\section{RESUMEN}

En este artículo se analiza el presente y futuro de la intermediación en el sector turístico, prestando especial atención en los tour operadores. El análisis se lleva a cabo después de años en los que ha habido toda una serie de importantes cambios en el sector, entre ellos el advenimiento de internet y las otras tecnologías de la información y comunicaciones (TIC). La aparición de internet ha reducido la necesidad de intermediación por un tour operador debido a que internet facilita la coordinación directa por el mismo turista y ha reducido también sus costes de búsqueda. A pesar de esto, sin embargo, es nuestra opinión que los tour operadores, aunque tal vez bajo distintas formas, seguirán estando presentes en la cadena de valor del producto turístico. Esto es así porque, en primer lugar, los tour operadores aún tienen una función importante a desarrollar en la cadena de valor turística; esto es, aportar reputación para resolver la problemática derivada de la incertidumbre respecto a la calidad a la que se enfrentan los consumidores, incertidumbre que no desaparece con internet. $\mathrm{Y}$, en segundo lugar, aunque internet facilita la operativa independiente por parte de los propios turistas potenciales, las TIC (internet inclusive) también incrementan la 
productividad de los intermediarios permitiéndoles ofrecer a los turistas un mayor número de servicios de mayor calidad.

Palabras clave: reputación, información asimétrica, costes de transacción, internet.

Clasificación JEL: L14, L15, L83

\section{INTRODUCCIÓN}

El objetivo de este artículo es analizar los cambios que está experimentando la intermediación en el sector turístico, cambios en los que tienen un papel central las tecnologías de la información y comunicaciones (TIC) en general, e internet en particular. Entre estos cambios podemos encontrar la creciente des-intermediación; el 'dynamic packaging'; la aparición de nuevos tipos de intermediarios on-line; la reciente fusión de los grandes tour operadores europeos: TUI y First Choice; y la de Thomas Cook y My Travel; etc. Así, desde el análisis económico, y aportando algo de evidencia empírica, el artículo plantea un análisis de presente y de futuro de la intermediación en el sector turístico, prestando especial atención al papel de los tour operadores. ${ }^{1}$ En este análisis de la organización de la cadena de valor del producto turístico, la importancia de internet y las demás TIC es y seguirá siendo enorme. Lo que no está tan claro es la intensidad del impacto de internet en la intermediación turística: ¿internet conducirá a la desaparición de la intermediación o, en cambio, comportará cambios sin llevar a su desaparición?

Para llevar a cabo nuestro análisis, vamos a empezar analizando y discutiendo cuál ha sido la función tradicional (pre-internet) de los tour operadores en la intermediación de la cadena de valor del producto turístico para, a continuación, analizar cuál será el impacto de internet y las otras TIC en la organización de la cadena de valor del producto turístico (el futuro de la intermediación).

1 Nuestro enfoque se centra en los operadores turísticos, a pesar de que la intermediación en el sector turístico es también llevada a cabo por las agencias de viajes. En nuestro análisis, se subsumen las agencias de viajes en función de los operadores turísticos, considerándolos como un solo interlocutor. Como se aclarará más adelante, puesto que no afecta el mensaje principal de nuestro trabajo, las agencias de viajes presentan mayor propensión que los operadores turísticos a que su papel sea des-intermediado en mayor medida por la presencia de internet (véase, por ejemplo, O'Connor y Frew, 2002). 
El producto turístico (sea o no comercializado mediante paquete que combina los diversos elementos que lo componen) consiste en el transporte (avión, tren, bus, etc.) al destino, el alojamiento en un hotel o apartamento, la comida, los servicios de entretenimiento, el entorno natural del destino, etc. Todos estos bienes y servicios definen lo que es el producto turístico, el cual, así pues, presenta dos características principales. En primer lugar, el producto turístico es un producto compuesto, una cesta compuesta de una variedad de distintos bienes y servicios, en lugar de un único bien o servicio. En segundo lugar, el producto turístico es un 'bien de experiencia' y no un 'bien de búsqueda', en términos de Nelson (1974). En un bien de experiencia la calidad del bien solamente es descubierta por el comprador después de su compra o consumo; en otras palabras, existe información asimétrica entre el vendedor y el consumidor respecto a la calidad del bien o servicio intercambiado. Estas dos características del producto turístico (bien compuesto, y bien de experiencia) han determinado el papel tradicional (pre-internet) desarrollado por los intermediarios del sector (tour operadores y agencias de viajes).

El tour operador tradicionalmente ha negociado con hoteles, empresas de transporte y otras empresas, combinando estos distintos elementos en el paquete turístico para venderlo al consumidor final, vía agencias de viajes, como un único producto y a un único precio (Sheldon, 1986). Así, un tour operador es una alternativa a lo que sería el intercambio directo en el mercado entre proveedores finales (hoteles, aerolíneas, etc.) y el consumidor final. Entonces, según Coase (1937), la existencia de los tour operadores y las agencias de viaje vendría determinada por el hecho que la intermediación reduciría los costes de transacción en los que se incurriría mediante el intercambio directo en el mercado. Más concretamente, los costes de transacción del intercambio directo entre consumidores finales y vendedores en el sector turístico consistirían en: (i) costes de coordinación; (ii) costes de búsqueda; y (iii) costes de incertidumbre respecto a la calidad. Así pues, la misión tradicional de los tour operadores y agencias de viajes ha sido la de facilitar la compra-venta al consumidor reduciendo, o incluso eliminando, estos costes de transacción en los que incurriría en caso de contratar directamente el producto turístico con los distintos vendedores finales.

Una vez identificada la tradicional (pre-internet) justificación de la existencia de operadores turísticos de acuerdo con la teoría de los costes de transacción de la empresa, debemos ser capaces de analizar el impacto de la llegada de internet en la industria. A pesar de que hace tiempo que la cadena de valor del producto turístico ha incorporado varios sistemas de las TIC (tales como los sistemas informatizados de reserva, CRS 'computer reservation system', y los sistemas de distribución global, GDS 'global distribution system') para organizar la relación 
entre varios de los diversos agentes como agencias de viajes y compañías de avión, internet ha supuesto un cambio importante dado que permite un acceso directo de los consumidores finales a los proveedores finales (Buhalis y Licata, 2002). Como consecuencia de ello, muchos profesionales y académicos han pronosticado la futura des-intermediación de la mayor parte de la cadena de valor del producto turístico mediante la que los consumidores finales serán capaces de saltarse a los intermediarios, ganando con ello debido a los menores precios que se producirán por una intensificación de la competencia. De hecho, existe evidencia de que un cierto grado de des-intermediación ya ha tenido lugar (The Economist, 2005), y es altamente probable que seguirá teniendo lugar en el futuro. Tal des-intermediación se explicaría (en la teoría de los costes de transacción de la empresa) por el hecho de que internet reduce tanto los costes de búsqueda como los costes de coordinación directa entre el turista y los vendedores finales, reduciendo así la necesidad de intermediación de un tour operador.

A pesar de ello, este artículo plantea dos hipótesis, ambas en la línea de resaltar el importante papel futuro que seguirán teniendo los intermediarios turísticos. En primer lugar, y a pesar que internet y las TIC en general facilitan la desintermediación, es decir, la compra directa de los turistas a los proveedores finales (hoteles, etc.), los intermediarios turísticos siguen y seguirán teniendo un papel primordial como proveedores de reputación, de información creíble a los turistas respecto a la calidad del producto final. Es decir, serán importantes en la resolución de la incertidumbre de calidad entre compradores y vendedores finales. Esto es así debido a que, a pesar de sus enormes posibilidades en el intercambio de información, la incertidumbre respecto a la calidad del producto no desaparece con internet, ni, por lo tanto, desaparece la necesidad de un intermediario en la cadena de valor. Consecuentemente, según nuestra opinión, en la era de internet persiste la justificación para la presencia de la intermediación en la cadena de valor turística en el suministro de información fidedigna y creíble al consumidor respecto a la verdadera calidad del producto turístico.

En segundo lugar, es también importante resaltar como internet y las TIC, además de facilitar el intercambio directo, tienen también un impacto importante en el funcionamiento de los intermediarios, convirtiéndolos en más eficientes y ampliando el tipo y la calidad de los servicios que pueden ofrecer. Esto, claro está, facilitará también la pervivencia de los intermediarios en la cadena de valor del producto turístico.

Este artículo no es el primero en ofrecer un análisis de los tour operadores turísticos. Por ejemplo, uno de los primeros es Sheldon (1986) que esboza algunas de las funciones que ofrecemos en este artículo. Sin embargo, la nuestra es la primera apor- 
tación en abordar teóricamente en el marco de la teoría de los costes de transacción la cuestión del impacto de internet sobre el papel de los tour operadores en la industria del turismo.

Es necesario tener en cuenta que aunque en nuestro artículo afirmamos que la intermediación en la cadena vertical del producto turístico seguirá siendo importante en la era de internet, no abordamos la cuestión de qué tipo de intermediario habrá. Es decir, ¿los operadores turísticos tradicionales, como por ejemplo TUI, seguirán dominando el mercado, o nuevos intermediarios on-line tomarán su lugar en la intermediación del producto turístico? Esta cuestión se aborda, por ejemplo, en Buhalis y Licata, 2002.

Por último, también es importante destacar que el propósito de este trabajo no es llevar a cabo un análisis de la organización industrial del sector de los operadores turísticos. Es decir, no tratamos aquí con cuestiones relativas a la interacción competitiva entre los diferentes operadores turísticos, la rentabilidad de la industria, estrategias de segmentación o la integración vertical hacia arriba o hacia abajo (véase, p. ej., Baum y Budambi, 1994; Renshaw, 1994; Aguiló et al., 2003).

El artículo se organiza de la siguiente manera. En la sección 2 llevamos a cabo una breve descripción del sector turístico y su evolución estos últimos años, prestando especial atención en el papel jugado por los tour operadores. En la sección 3, a partir de la teoría de los costes de transacción, analizamos las funciones que llevan a cabo los tour operadores, mientras en la sección 4 analizamos el impacto de internet en la organización de la cadena de valor del producto turístico. En la sección 5 discutimos como internet también ha tenido impacto en la eficiencia de los tour operadores y en los servicios que estos pueden ofrecer, y concluimos en la sección 6 .

\section{UNA MUY BREVE DESCRIPCIÓN DEL SECTOR Y LA INTERMEDIACIÓN TURÍSTICA}

Mirando retrospectivamente, parece clara la importancia del papel que han tenido los intermediarios en el enorme desarrollo del sector turístico español, uno de los principales destinos turísticos mundiales. ${ }^{2}$ La llegada masiva de turistas extranjeros a España que tuvo lugar a partir de los 60s (principalmente, turismo vacacional, en verano) se realizó,

2 España es el segundo destino turístico mundial medido en función de las llegadas de turistas extranjeros: en el año 2005 España recibió 55,8 millones de turistas (World Tourism Organization, 2006). 
en gran medida, mediante el concurso de los tour operadores europeos. Así, los turistas europeos que venían a España compraban paquetes vacacionales (package tour) a agencias de viajes, las cuáles vendían los paquetes que les distribuían los tour operadores.

Los tour operadores llevaban a cabo el diseño y la organización de este paquete turístico mediante la compra de alojamiento a los establecimientos de las distintas regiones turísticas, el transporte aéreo mediante el flete de vuelos chárter, etc.. Los tour operadores europeos incluso tuvieron un papel importante en el desarrollo de la oferta de alojamiento en España, por ejemplo en las Baleares. En algunos casos, los tour operadores financiaron la construcción de establecimientos hoteleros con el compromiso de disponer de dicha capacidad de alojamiento para su distribución los siguientes años en los mercados europeos.

Así, la cadena vertical de la industria turística estaba muy bien definida y era imposible saltarse ningún paso; en otras palabras, la desintermediación no era posible: el consumidor final o turista adquiría su paquete turístico a intermediarios al por menor (agencias de viaje) que adquirían estos paquetes a intermediarios al por mayor (tour operadores) que eran quiénes contrataban los bienes y servicios finales del producto turístico a compañías hoteleras y aerolíneas.

Los paquetes turísticos que se comercializaban eran paquetes turísticos estándares. Básicamente había dos posibilidades, viajes o paquetes de 7 días o de 14 días en destinos que pudieran proveer sol y playa, para una demanda concentrada mayoritariamente alrededor de los meses de verano.

\subsection{Cambios en el sector y en la intermediación turística}

A partir de la situación descrita en el apartado anterior, en estos últimos años han tenido lugar una gran cantidad de cambios en la industria del turismo en general, y en la intermediación en particular. Todos estos cambios han estado relacionados, directa o indirectamente, con la aparición y el impresionante desarrollo de internet y de las otras TIC.

Globalización. El sector turístico no se ha escapado, más bien al contrario, del proceso de globalización que ha experimentado la economía mundial estos últimos años. Entre muchos otros aspectos, ha tenido lugar la aparición de nuevos destinos turísticos de primer orden; la internacionalización de las empresas turísticas (por ejemplo, la cadena hotelera Sol Melia); etc.

Low cost. Otro fenómeno que ha tenido un gran impacto en el sector turístico en general es la irrupción en el transporte aéreo de las low cost, o aerolíneas de bajo cos- 
te. A modo de ejemplo, en España, en el año 2006, el $31.6 \%$ de pasajeros vía aérea llegan ya en una compañía de bajo coste, según INESTUR. Este cambio, junto con el cambio general del sector de las aerolíneas, ha supuesto la (casi) total desaparición de los vuelos chárter.

Internet y TIC. Internet y las TIC (ordenadores, servidores, plataformas web, etc.) han supuesto un gran cambio per se, además de estar relacionado con el proceso de globalización y la aparición de las low cost. Internet facilita las relaciones tanto con los clientes (B2C) como con los proveedores (B2B). Así, internet abarata los costes de distribución, de manera especial para las empresas grandes (por ejemplo grandes cadenas hoteleras). Esto es así porque una plataforma web requiere una inversión muy importante (en tecnología, líneas de comunicación, hardware, etc.) lo cual implica que para que sea rentable es necesaria una escala de negocio mínima.

\subsection{Tipos de viajes y paquetes turísticos.}

Todos estos factores, junto con otros, han cambiado en gran manera el tipo de viajes y de paquetes turísticos comprados por los turistas tanto extranjeros como españoles. Antes, como hemos apuntado más arriba, gran parte de los viajes y paquetes turísticos consistían en viajar a destinos vacacionales mediante paquetes turísticos estándares de una o dos semanas, paquetes que desarrollaban los tour operadores y que se comercializaban mediante las agencias de viaje, y en los que se incluía todos los elementos más importantes del viaje (alojamiento, transporte).

Ahora, debido a que los costes fijos de viajar se han reducido sustancialmente, con el papel predominante jugado por las low cost, la gente realiza viajes más cortos, y hace más viajes a lo largo de todo el año, incluyendo también una variedad mayor de destinos. Esto, por lo tanto, implica un paquete (en el caso en que el viaje se lleve a cabo mediante paquete turístico) más adaptado a las nuevas formas de viajar, incluyendo la posibilidad por parte del turista de comprar on-line parte del viaje o paquete turístico a un intermediario y, otra parte, directamente a los proveedores finales.

\section{LA TEORÍA DE LOS COSTES DE TRANSACCIÓN Y LOS TOUR OPERADORES}

La cuestión básica que analizamos y discutimos en este artículo está directamente relacionada con la pregunta de porqué hay intermediación por los operadores de viajes 
en la cadena de valor del producto turístico, en lugar de que los consumidores finales o turistas compren ellos mismos todos y cada uno de los distintos componentes del producto turístico (alojamiento, transporte, etc.) a los vendedores finales. La lógica tras la intermediación en la cadena de valor del producto turístico nos permitirá estudiar el impacto de las TIC, y más específicamente, internet en la organización de la cadena de valor. En un primer momento, vamos a abstraer internet de nuestro análisis y vamos a analizar la intermediación en la cadena de valor del producto turístico, centrando el análisis en la organización de esta cadena en la era previa a internet. Luego, en el siguiente apartado, discutiremos el impacto que internet y otras TIC han tenido, y tendrán en el futuro.

Un tour operador es una empresa que negocia con hoteles, empresas de transporte, y otros suministradores, y combina estos componentes de vacaciones en un paquete turístico. Este paquete turístico (la combinación de los componentes de unas vacaciones, tales como el alojamiento, el transporte, entretenimiento, comidas, etc.) se vende después al consumidor final como un único producto a un único precio (Sheldon, 1986). Así, el tour operador intermedia entre los ofertantes de los distintos productos y servicios que por definición conforman el producto turístico, y el consumidor final o turista ${ }^{3}$.

FIGURA 1.-El tour operador como un intermediario

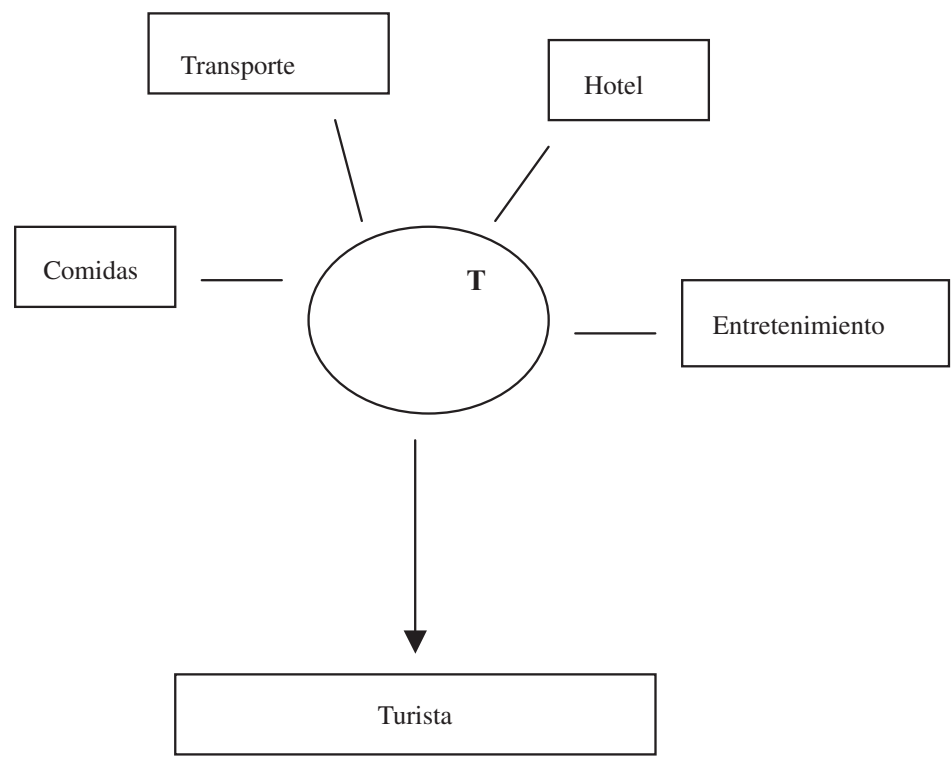

${ }^{3}$ Tal y como dijimos en la introducción, hacemos abstracción de las agencias de viaje. 
En este artículo, nos aproximamos al análisis del papel y la presencia de los tour operadores en la cadena de valor turística mediante la teoría de los costes de transacción (Coase, 1937; Williamson, 1975). El punto principal de la teoría de los costes de transacción consiste en preguntar porqué existen las empresas, en lugar de haber solamente mercados ${ }^{4}$. Es decir, porqué algunas transacciones (por ejemplo, la producción de un determinado input necesario para una empresa) se llevan a cabo dentro de las empresas, mientras otras se llevan a cabo mediante el intercambio en el mercado (la compra a un proveedor). La respuesta que aporta la teoría yace en el reconocimiento de que tanto las transacciones internas en una empresa como las transacciones en el mercado conllevan unos costes de transacción. Estos costes de transacción son los costes de coordinar las distintas actividades necesarias para llevar a cabo la transacción, los costes de motivar a las personas y a las organizaciones implicadas, el coste de contratar y renegociar, los costes de transportar el bien, etc. Además, a medida que la dificultad de la transacción aumenta (por ejemplo, debido a una mayor asimetría informativa entre las partes) los costes de transacción aumentan y, entonces, el excedente social de la transacción disminuye.

La teoría de los costes de transacción predice que cualquier transacción se llevará a cabo, se organizará dentro de una empresa siempre que sea eficiente, es decir, siempre y cuando los costes de internalizar la transacción sean menores que los costes de organizar la transacción en el mercado (y viceversa); o, en otras palabras, siempre que el excedente social de internalizar la transacción sea mayor que el de usar el mercado. Además, la teoría de los costes de transacción afirma que para transacciones difíciles, los costes de transacción son menores cuando estos tienen lugar dentro de la empresa en lugar de a través del mercado; es decir, el excedente social de llevar a cabo la transacción dentro de los límites de la empresa en lugar de mediante el mercado es mayor cuando la dificultad de la transacción incrementa. En la siguiente figura 2 ilustramos esta idea (Gibbons, 2000).

${ }^{4}$ Como cuestión de hecho, hay una amplia gama de modos de gobierno intermedio entre el mercado y la empresa, tales como joint-ventures, contratos a largo plazo, etc. Su análisis en la industria del turismo está fuera del objetivo de este artículo. 
Figura 2.-Empresa versus mercado

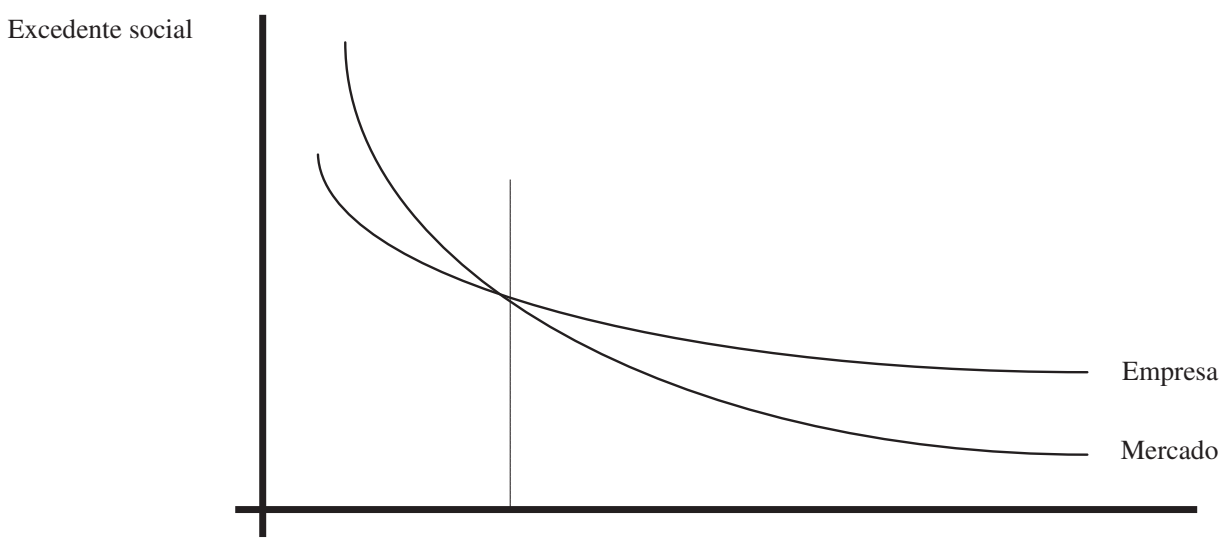

Dificultad de la transacción

Aplicando el marco de la teoría de los costes de transacción al análisis de la organización de la cadena de valor del producto turístico, necesitamos comparar los costes de transacción que aparecen cuando un turista trata directamente mediante el mercado con los vendedores finales (hoteles, aerolíneas, empresas de transporte de autobús, etc.), versus los costes de transacción que aparecen cuando un tour operador intermedia y, por lo tanto, empaqueta todos los bienes y servicios que componen el paquete turístico y lo vende al turista o consumidor final. La teoría de los costes de transacción indica que la compra se llevará a cabo mediante el tour operador cuando sea más eficiente (menores costes de transacción) que no que el consumidor compre de manera directa el producto turístico a los múltiples vendedores finales ${ }^{5}$.

5 En todo el análisis del artículo subyace el supuesto de la incompletitud de los contratos (Tirole, 1999). Es difícil (demasiado costoso en general) prever y especificar en el contrato entre las partes todas las contingencias futuras pertinentes; los contratos dejan muchas cosas sin considerar, y están abiertos, son ambiguos en su interpretación; por todo esto, los contratos no son las herramientas perfectas capaces de administrar con precisión el desarrollo de una transacción. Y ésta es precisamente la razón por la que la organización de la cadena de valor turística es importante; la organización que minimice el comportamiento oportunista y los costes de transacción de cualquier tipo, será la organización que debemos esperar obtener. 
Cuando analizamos la cadena de valor turística, observamos que los costes de organizar las transacciones mediante el mercado consisten $\mathrm{en}^{6}$ :

- Costes de búsqueda

- Costes de coordinación

- Costes de incertidumbre en la calidad

\subsection{El tour operador como coordinador}

El producto turístico consiste en una serie de bienes y servicios cuyo consumo requiere de una ajustada coordinación entre ellos. Tal y como afirma Inbound Holiday para el Gold Coast: «services include the coordination of all ground arrangements including hotels, motels, executive apartments, farm stay, airport transfers with meet \& greet services, coach charter, stretch limousines, restaurants, golf, tours, car rental, cruises, interpreters, tour guides, educational tours for overseas students and conventions.»

Todos estos, y posiblemente otros servicios y bienes conforman el producto turístico, y para que el producto turístico aporte alta utilidad (satisfacción) al consumidor, debe existir una coordinación ajustada de todos estos bienes y servicios a lo largo de distintas dimensiones, especialmente en lo que concierne a la dimensión cronológica. Cualquier fallo de coordinación a lo largo de estas líneas implica una pérdida de utilidad para el turista. Además, en caso de un fallo de coordinación, ésta debe de arreglarse rápidamente con tal de controlar los daños causados al turista y que éste pueda disfrutar de unas vacaciones razonables.

Cuá es la forma eficiente de coordinar todos (o algunos de) estos componentes del producto turístico? Una posibilidad es que el turista mismo se encargue de contratar con todos y cada uno de los vendedores finales (hoteles, etc.) y se asegure la coordinación contratando las características requeridas y las condiciones de cada uno de los bienes y servicios. Sin embargo, puesto que los contratos son incompletos, este mecanismo de coordinación será claramente imperfecto y susceptible de conllevar importantes errores.

${ }^{6}$ En este artículo dejamos de lado algunas cuestiones que, aunque importante en la teoría de los costes de transacción, a nuestro juicio no son cruciales en el análisis del papel que desempeñan los operadores turísticos, como por ejemplo, la especificidad de los activos y la posibilidad de expropiación de las inversiones. 
De hecho, la coordinación de los distintos componentes (bienes y servicios) del producto turístico tiene lo que Milgrom y Roberts (1992, p. 117) llaman 'atributos de diseño': «los problemas de coordinación en los que a priori existe información sobre cómo las partes de la decisión deben ajustarse entre sí, y en dónde los pequeños errores son muy costosos, se dice que tienen atributos de diseño». Entonces, según Milgrom y Roberts (1992), «un diseño centralizado de las variables de diseño tiende a reducir tanto el coste de los errores como la cantidad de comunicación y búsqueda necesarias para identificar una decisión óptima»

En nuestro marco esto implica que la coordinación centralizada por el tour operador asegura un resultado más eficiente, incluyendo una mejor resolución de los posibles errores de coordinación que pudieran surgir durante las vacaciones. Así, la intermediación mediante un tour operador, probablemente resuelve de una manera más eficiente los requisitos de coordinación del producto turístico, en lugar de confiar en que sea el consumidor final el que intente coordinar por si mismo todas las partes del producto turístico acudiendo a todos los vendedores finales y estableciendo un conjunto de contratos privados con todos ellos.

\subsection{El tour operador y la búsqueda de información por parte del turista}

Para la obtención de información sobre precios, calidad y el resto de características del producto turístico, los consumidores finales o turistas deben llevar a cabo un proceso de búsqueda costoso ${ }^{7}$. El coste de la búsqueda comprende el dinero, el tiempo y el esfuerzo que los potenciales compradores invierten en obtener la información sobre los precios, las características y las calidades para el alojamiento, vuelos, etc. Ejemplos de tales costes de búsqueda de información son las visitas a las agencias de viaje, llamadas telefónicas, comprar periódicos, etc.

Tal y como vemos en la figura 3 (obtenida de Whinston, Stahl y Choi, 1997), la búsqueda individual de cada consumidor generará muchas duplicidades de esfuerzo y de costes de búsqueda. Cada turista potencial debe visitar o llamar a cada vendedor final potencial (hotel, aerolínea, empresa de autobuses, etc.) e incurrir en el coste (tiempo)

7 Aquí nos centramos en la organización del proceso de búsqueda, y no en las consecuencias en términos de, por ejemplo, los precios resultantes de tales costes del proceso de búsqueda (véase Carlson y McAfee, 1983). 
de buscar y recolectar la información. Tal duplicación del esfuerzo sería minimizada mediante el uso de intermediarios tales como los tour operadores y las agencias de viajes las cuales recogen, procesan y almacenan toda la información relevante.

FIGURA 3.--Intermediación o desintermediación en la búsqueda de información

Des-intermediación

Consumidor
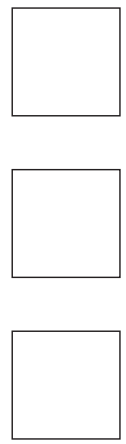

Vendedor

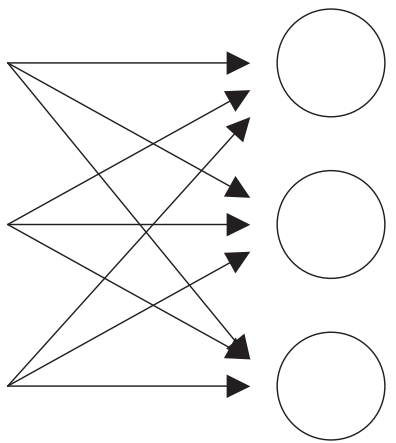

Consumidor

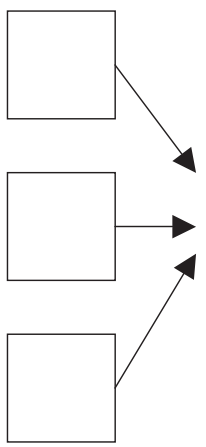

Intermediación

Vendedor

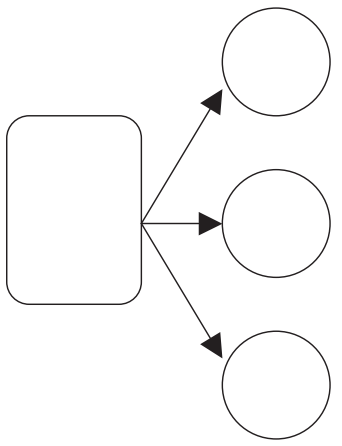

El papel de los tour operadores y las agencias de viaje en el incremento de la eficiencia en el proceso de búsqueda de información resulta simplemente de su papel de aportar un lugar centralizado para todos los vendedores. Además, esto no implica que exactamente la misma información sea proporcionada por los tour operadores que por los mismos vendedores (hoteles, aerolíneas, etc.). En lugar de esto, la información sobre el producto turístico disponible en el intermediario será solamente la necesaria para que los compradores o turistas potenciales puedan tomar sus decisiones de compra. Así, los tour operadores y las agencias de viajes también filtran información, lo cual incrementa aún más la eficiencia del proceso de búsqueda cuándo es intermediado.

A pesar de toda la información que los consumidores reciban de los vendedores previamente a la compra del producto, aún tienen que confiar en lo que los vendedores anuncian sobre sus producto. A menudo, las empresas vendedoras (hoteles, aerolíneas, etc.) puede que no aporten toda la información mediante la cuál los consumidores puedan evaluar sus productos o servicios; o que la información sea inadecuada para juzgar si los vendedores son empresas de confianza o no. Por todo esto, el papel de los tour 
operadores en el mercado de información va más allá de ser un simple depositario de la información aportada por los vendedores finales. Éste es el tema que discutimos en la sección siguiente.

\subsection{El tour operador y la incertidumbre respecto a la calidad}

La teoría económica distingue entre 'bienes de experiencia ' y 'bienes de búsqueda ‘ (Nelson, 1970). Bienes de búsqueda son aquellos bienes o servicios cuya calidad puede ser conocida por los consumidores antes de su consumo y, por lo tanto, antes de la compra. Para los bienes de experiencia, en cambio, su calidad sólo se conoce durante o después del consumo del bien o servicio. El producto turístico, como la mayoría (si no todos) de los servicios, es un bien de experiencia. La calidad del servicio en un hotel sólo puede determinarse durante la estancia, la calidad de un vuelo (puntualidad en la hora de salida, etc.) sólo se conoce durante el vuelo en sí, la calidad de la comida es otro ejemplo de bien de experiencia, y lo mismo para todos los otros bienes y servicios que componen el producto turístico.

Así pues, una cuestión crucial es la incertidumbre a la que se enfrenta el turista respecto de la verdadera calidad del producto turístico antes de su consumo, es decir, antes de las vacaciones en sí. En un lenguaje algo más técnico, existe asimetría de información entre el vendedor (hotel, compañía aérea) y el comprador del servicio turístico, ya que el vendedor tiene una mejor información que el comprador o turista en relación a las características específicas y la calidad del producto turístico que se intercambia.

La literatura económica muestra que una consecuencia de tales asimetrías de información puede ser un fallo del mercado: una transacción que sería a priori beneficiosa, generadora de valor para todas las partes, no se llevaría a cabo. Esto es lo que podría ocurrir en una situación de selección adversa (definida como aquella en la que el vendedor tiene una información superior a la del comprador antes de la firma del contrato), descrita por primera vez por Akerlof (1970) ${ }^{8}$. Akerlof demostró que en los mercados con información asimétrica entre vendedores y compradores (por ejemplo, en los mercados de automóviles de segunda mano), la selección adversa podría llevar a que los bienes de mala calidad expulsaran del mercado a los de buena calidad. Debido a que el comprador tiene incertidumbre respeto de la calidad del bien, teme que éste sea uno de

${ }^{8}$ El riesgo moral, en cambio, se da cuando existe información asimétrica después de firmar el contrato (Holmstrom, 1979). 
baja calidad y por lo tanto solamente está dispuesto a pagar un precio bajo por él. Esto, a su vez, hace que las empresas con buenos productos no estén dispuestas a vender. Como consecuencia de ello, sólo existirá un mercado para los productos de baja calidad $\mathrm{y}$, por lo tanto, intercambios potencialmente generadores de valor, como los de bienes de alta calidad, puede que no se lleven a cabo.

Dada la existencia de esta asimetría de información entre el vendedor o vendedores y el comprador, y el consiguiente posible fallo de mercado, la cuestión consiste en qué se puede hacer al respecto. La solución obvia es que los vendedores del producto turístico informen - y convenzan - a los compradores potenciales sobre la calidad real del producto, eliminando así la asimetría de la información. Existen varios mecanismos posibles para ello, entre los cuales podemos encontrar: (i) la publicidad (Nelson, 1974), (ii) el establecimiento de estándares de calidad por parte de la misma industria o por el gobierno, (iii) utilizar el precio como una señal para la calidad (Wolinsky, 1983), y (iv) la utilización de intermediarios como canal para aportar información creíble.

Todos y cada uno de estos mecanismos desempeñan algún papel en la resolución de la incertidumbre existente sobre la calidad entre los vendedores finales y los turistas. Nosotros, sin embargo, nos centramos en el que creemos que es uno de los principales mecanismos para proporcionar una solución al potencial fallo de mercado debido a las asimetrías de información: el papel jugado por un intermediario. Cualquiera de los mecanismos mencionados es costoso - es decir, los costes de transacción se incrementan debido a la asimetría de información entre vendedores y compradores, y debido a la necesidad de proporcionar una solución. Nuestra opinión es, sin embargo, que la intermediación de un tour operador puede ser eficiente ya que puede proporcionar una solución de bajo coste para el problema de la asimetría de información entre las empresas hoteleras y otros vendedores finales y los turistas.

Un tour operador (un intermediario, en general), mejora la eficiencia del mercado al ofrecer información fidedigna sobre la calidad del producto. De esta manera, se elimina la posibilidad de un fallo de mercado debido a la incertidumbre de calidad, y lo hace con costes de transacción menores que los de los posibles mecanismos de organización alternativos en los que el turista contrata directamente con los vendedores finales.

El papel a jugar por un intermediario puede ser de dos tipos. Un intermediario puede ser un 'experto' que ha adquirido los conocimientos y las habilidades adecuadas para evaluar la calidad del bien o servicio en cuestión (Biglaiser, 1993). Este tipo de intermediación es especialmente importante en entornos en los que el uso y el consumo del bien no son suficientes para evaluar la calidad de un bien; por ejemplo, algunos minoristas evalúan la calidad de un reproductor de DVD. Parte de este papel de los expertos es interpretado por los tour operadores en la industria turística, por ejemplo, 
algunos tour operadores evalúan y realizan un seguimiento de la gestión medioambiental de los establecimientos hoteleros (por ejemplo, Orizzonti, un tour operador italiano, Japan Travel Bureau, Scandinavian Leisure Group y TUI).

Sin embargo, probablemente el principal papel de los tour operadores se deba a la capacidad del tour operador de construirse y labrarse un nombre de marca y una reputación de vender y comercializar paquetes turísticos de alta calidad. En este caso, la intermediación puede aumentar la eficiencia del mercado, incluso en el caso en el que los intermediarios no tengan un mayor conocimiento ni mayores habilidades para evaluar la calidad (no sean «expertos»). Los tour operadores serían una fuente de información de la calidad simplemente por su reputación de proporcionar productos de alta calidad. Veamos a continuación cómo funciona este mecanismo.

\subsubsection{La reputación y la repetición de las compras}

Un método convencional para hacer frente a la incertidumbre de calidad es la construcción de la marca y la reputación de un vendedor específico. La cuestión clave para construir una reputación de ofrecer productos de alta calidad es que la empresa sea un jugador de largo plazo o que el producto sea comprado en varias ocasiones. Mientras que en una interacción de una sola transacción la empresa tiene grandes incentivos a mal informar respecto de la calidad al comprador potencial, en un marco de interacción repetida con la empresa puede tener un interés en el mantenimiento de una sólida reputación con el fin de asegurar futuros negocios (Kreps y Wilson, 1982).

El mismo incentivo para construir una buena reputación puede aparecer en caso de que la empresa interactúe en varias ocasiones con diferentes compradores - en este caso, la reputación se pasa a lo largo de los consumidores. Para los productos o servicios que se utilizan sólo una vez, la reputación se construye sobre la empresa más que sobre el producto y la reputación específica de la empresa se convierte en la marca con la que transfiere confianza a los consumidores producto a producto. Además, un requisito básico para cualquier sistema de reputación es que cada parte, tanto el vendedor como el comprador, debe ser capaz de evaluar la calidad correctamente después de la compra, es decir, evaluar el comportamiento pasado de la otra parte.

Así, cuando la interacción entre el vendedor y el comprador no es frecuente o, cuando es difícil para una persona ajena a la operación determinar la calidad del bien que se intercambia, la construcción de una reputación es difícil o imposible. Y este es exactamente el caso de la industria turística en un intercambio directo entre los vendedores finales (por ejemplo, hoteles) y los turistas. Téngase en cuenta la forma en que, por 
ejemplo, una empresa hotelera, podría construir una reputación de ofrecer servicios de alta calidad. Muchas veces, la interacción entre un turista y un establecimiento hotelero se produce sólo una vez, ya que los turistas cambian de destino o lugar de alojamiento con bastante frecuencia. ¿Cuál es entonces el incentivo de una compañía hotelera a proporcionar y mantener una alta calidad del servicio? Dado que es poco probable que el turista repita y que para los turistas potenciales es difícil saber si la calidad ha sido alta o baja, el incentivo para ahorrar suministrando baja calidad es alto con lo cual, el vendedor final en la industria turística no tiene muchos incentivos para proporcionar alta calidad.

Un tour operador, en cambio, tiene un doble incentivo a mantener una buena reputación. En primer lugar, con el turista. El tour operador va a proveer paquetes turísticos para diferentes destinos y muchos consumidores finales son potenciales repetidores frente a los que tiene incentivos a asegurar que la calidad será la prometida para no perderlos. Y en segundo lugar, con el vendedor final. El tour operador es un comprador repetidor al que el vendedor final tiene incentivos a mantener la calidad adecuada. En caso contrario, que la calidad no sea suficientemente alta, el tour operador dejará de distribuir el producto de este vendedor final para mantener su reputación ante los consumidores finales.

Tres salvedades de los análisis anteriores respecto del papel de los operadores turísticos se aplican a las grandes empresas, a las empresas (del destino) con una alta tasa de visitantes que repiten, así como a empresas de baja calidad. Los grandes vendedores (por ejemplo, las grandes cadenas hoteleras como Hilton) pueden encontrar fuertes incentivos para construir una reputación y un nombre de marca de calidad: un turista cualquiera tiene la opción de alojarse en un establecimiento de la cadena en muchos destinos diferentes y la probabilidad que un comprador final o turista repita en algún establecimiento de la cadena es alta. Como consecuencia, una gran cadena hotelera tiene incentivos a mantener una reputación de alta calidad. La misma salvedad para la construcción de la reputación se puede aplicar a (pequeñas) empresas de un destino turístico donde los turistas tienen una alta tasa de repetición. Cuando la repetición de los compradores finales o turistas es alta, incluso una pequeña empresa puede mostrar niveles elevados de calidad si se espera que una alta proporción de los turistas tiendan a volver en un futuro próximo. Y, por último, y de manera intuitiva, las empresas de baja calidad no tienen necesidad de un operador turístico para certificar una calidad baja. Tal y como se explica en el ejemplo del mercado de coches de segunda mano en Akerlof (1970), el intercambio de productos de baja calidad no se ve obstaculizado por la existencia de información asimétrica. 


\section{INTERNET Y LA (DES)INTERMEDIACIÓN EN EL SECTOR TURÍSTICO}

Hace ya tiempo que el sector turístico, y más concretamente sus intermediarios, han incorporado en su quehacer las tecnologías de la información y las comunicaciones, como por ejemplo los 'computer reservation systems', CRS, y los 'global distribution systems', GDS. Sin embargo, la aparición y desarrollo de internet ha supuesto un cambio de muchísima mayor importancia en la organización de la cadena de valor del sector turístico al permitir el acceso directo de los consumidores finales o turistas a los proveedores finales como los hoteles. Así, según varios análisis, esta aparición de internet implicaría una futura desintermediación de la cadena de valor del sector turístico debido a que, por un lado, la coordinación directa por los mismos consumidores finales de los distintos bienes y servicios que componen el producto turístico es mucho más sencilla (aunque no a coste cero), así como, por otro lado, también los costes de búsqueda se han reducido enormemente gracias a internet y otras TIC. La búsqueda on-line ofrece tremendas ventajas por encima de la búsqueda física: en el ordenador y con los programas de búsqueda (Google) los consumidores pueden buscar en todo el espacio virtual a prácticamente coste cero en términos de tiempo y transporte, permitiendo procesar una gran cantidad de información adicional al precio, por ejemplo, localización y nombre de los vendedores, y características diversas del producto y del vendedor. Además de los menores costes de búsqueda, internet y las otras TIC permiten también a los consumidores recordar y comparar toda la información obtenida (Buhalis y Licata, 2002; Evans y Wurster, 2000; O'Connor y Frew, 2002). ${ }^{9}$

Efectivamente, los datos de diversas fuentes y encuestas respecto a la realidad española nos muestran esta doble tendencia (presente también en el resto de países): un mayor uso de internet (en reserva, pago, etc.), así como un menor peso porcentual de viajes con paquetes turísticos contratados a un tour operador. En primer lugar, los datos del INESTUR nos muestran como el uso de internet en la planificación del viaje (información, consultas, reservas, pagos) es importante, representando el año 2006 un $12,5 \%$ del total para el turismo interior español, y un 37,4\% para viajes turísticos con destino en el extranjero.

${ }^{9}$ En internet, la problemática puede aparecer no debido a la escasez de información o a la dificultad de su búsqueda, si no debido a la excesiva abundancia de información. En este caso, un intermediario puede tener la función de filtrar la información de manera que el consumidor solamente acceda a la información relevante. 
En segundo lugar, y respecto a la forma de organización del viaje, se observa como en el año 2001, el $50 \%$ de los turistas no residentes organizó su viaje a España mediante paquete turístico, mientras que en el año 2006 solamente fueron el $34 \%$ de los turistas no residentes los que vinieron mediante paquete turístico según el Instituto de Estudios Turísticos (Frontur). En la misma línea, y por lo que respecta al gasto turístico, mientras el año 2003 el 50\% del gasto total de los turistas se realizó por aquellos que usaron paquete turístico, en el año 2006 este porcentaje se redujo al 35\% (Instituto de Estudios Turísticos, Encuesta de gasto turístico). Así pues, se observa una clara tendencia a la reducción del uso del paquete turístico en la organización del viaje.

Nuestro enfoque aquí es examinar en qué medida el papel de los intermediarios en la reducción de los costes de búsqueda, en el aumento de la eficiencia de la coordinación entre los diversos componentes del paquete turístico, y en el suministro de información veraz y fidedigna sobre la calidad de los diversos componentes del producto turístico, se ve afectada por la aparición de internet. A raíz de la teoría de los costes de transacción que se ha expuesto anteriormente, podemos evaluar en qué medida los costes de transacción de cualquiera de las alternativas organizativas (intercambio directo en el mercado, intermediación por un tour operador) se ven afectadas por internet y, así, de qué manera se ve afectada la estructura organizativa óptima.

\subsection{El tour operador como coordinador}

Según Davidow y Malone (1992), internet y las otras TIC han dado lugar a la empresa virtual, organización de la actividad económica en la que cada actividad de la cadena de valor es realizada por una empresa independiente, una empresa des-integrada, conectada a través de las relaciones de intercambio de mercado. Tal organización deviene posible gracias a la coordinación de los intercambios de mercado entre entidades independientes que permiten internet y las otras TIC.

Análogamente, en nuestro marco, la industria turística, internet ha facilitado la coordinación directa por parte del consumidor final o turista de los diferentes elementos que componen el producto o paquete turístico finalmente consumido. Así, el consumidor final o turista sí puede coordinar los horarios de vuelo con el alojamiento en el destino, las fechas de alquiler de vehículo o otros transportes, la compra de entradas para un espectáculo en la ciudad de vacaciones, o el transporte desde el aeropuerto de destino al hotel. Por lo tanto, una estrecha coordinación se ha vuelto más fácil (aunque no exenta de costes) y, por lo tanto, la necesidad de la intermediación de un tour operador para coordinar se ha reducido significativamente. 


\subsection{El tour operador y los costes de búsqueda de información}

La búsqueda on-line de productos y servicios turísticos ofrece una gran ventaja sobre la búsqueda física. Para empezar, mediante el uso de las TIC, tales como los motores de búsqueda, los consumidores pueden buscar en todo el espacio de información a un coste muy bajo en términos de tiempo y transporte. Además, la búsqueda on-line permite que los consumidores (turistas potenciales) procesen una amplia cantidad de información más allá de los precios - por ejemplo, la ubicación y el nombre de los proveedores, condiciones de venta, y otras características del producto. Aparte de estos bajos costes, también permite a los consumidores comparar la información obtenida de varios espacios de venta virtual ${ }^{10}$.

Como consecuencia de ello, los consumidores no necesitarían intermediarios para la búsqueda y habría una clara tendencia a la des-intermediación por la que el papel de los tour operadores en el proceso de búsqueda desaparecería. Sin embargo, a pesar de la importancia de internet en la reducción de los costes de búsqueda, aún hay al menos dos razones que pueden justificar, al menos parcialmente, la existencia de intermediarios en el proceso de búsqueda. Uno de ellas es que la duplicación del tráfico de la información, como se explicó anteriormente (ver figura 3) aún persiste, y la intermediación del tour operador cobra sentido por cuanto la búsqueda entre los intermediadores reduce drásticamente la duplicación del tráfico y refuerza la eficiencia de la red de información. La otra es que en internet, la desventaja de la limitación física se reemplaza con el difícil problema de localizar y procesar la información relevante, no a causa de la falta de información sino por la abundancia de ella. En este caso, un operador turístico (un intermediario) todavía puede desempeñar un papel en la filtración de información, de manera que los consumidores accedan sólo a la información relevante.

${ }^{10}$ Una alternativa a la búsqueda de los consumidores es la publicidad on-line de los vendedores finales. Sin embargo, aún no está clara su eficacia en comparación con la búsqueda de los consumidores. 


\subsection{Calidad e información asimétrica}

A pesar del importantísimo papel de internet y las TIC en el proceso de búsqueda de información, la eficiencia de la búsqueda dependerá de cuánta información los vendedores pongan disponible, y de la credibilidad de la información proporcionada. Así, a pesar de la abundancia de información, y de los bajos costes de búsqueda, el problema de la información asimétrica entre los vendedores y los compradores en el sector turístico no desaparece con internet. Con internet, es también difícil (costoso) para un comprador evaluar plenamente la calidad de los bienes y servicios que compra, y la información recibida puede ser insuficiente para juzgar si los vendedores son empresas con reputación (de fiar) o no. Así, sin ninguna garantía sobre la información y remedios disponibles para el caso en que haya disputas sobre la calidad del servicio, la reputación es también importante en la era de internet (Huston y Spencer, 2002; Melnik y Alm, 2002, 2005).

Tal y como hemos explicado más arriba, un tour operador (y una agencia de viajes) es capaz de construirse una reputación y una marca que aporte una señal de calidad a los turistas, incrementando así la eficiencia informativa, tanto en calidad como en contenido. Así, en la era de internet y las TIC, uno de los principales papeles de los intermediarios turísticos en la cadena de valor del sector turístico es, y será, la transmisión de información creíble y de fiar. Un primer trabajo que analiza el papel de los intermediarios turísticos en la transmisión de información es Clerides et al. (2006). En este trabajo se lleva a cabo un análisis explícito de los rankings ofrecidos por los tour operadores de los hoteles que ellos mismos distribuyen en contraposición a los rankings oficiales como el número de estrellas. En su análisis muestran como, efectivamente, la información aportada por los tour operadores es significativamente distinta a la que proporciona el número de estrellas (el ranking oficial) del hotel en cuestión.

\subsubsection{Intermediación en las Islas Baleares e incertidumbre respecto a la calidad.}

En Calveras y Orfila (2006) se analiza empíricamente si los intermediarios turísticos (tour operadores y agencias de viajes) desarrollan este papel de transmisión de reputación en la resolución de la información asimétrica respecto a la calidad entre los vendedores (establecimientos hoteleros) y compradores (turistas), y los resultados del análisis empírico concuerdan con la hipótesis del papel de los intermediarios como 
transmisores de reputación y de información creíble y veraz a los consumidores. El análisis empírico se lleva a cabo mediante una muestra representativa de establecimientos hoteleros que operan en las Islas Baleares, uno de los principales destinos turísticos del Mediterráneo y del mundo entero ${ }^{11}$.

En el trabajo de Calveras y Orfila (2006), se observa en primer lugar como el papel de los intermediarios turísticos en la distribución de la capacidad de alojamiento de un establecimiento turístico es mayor para los establecimientos de alta calidad que para los de baja calidad. Este resultado permite intuir que los problemas derivados de la información asimétrica son importantes para los hoteles de alta calidad, mientras que son de menor importancia para los hoteles de baja calidad (como en el caso del mercado de los coches de segunda mano). En segundo lugar, también se observa como los establecimientos que forman parte de una gran cadena hotelera utilizan en menor medida los servicios de un intermediario. ${ }^{12}$ Esto sería así porque las grandes cadenas hoteleras son capaces de construir su propia reputación y marca, con lo que no necesitan de un intermediario para señalar su calidad. ${ }^{13}$ Finalmente, se muestra como el análisis empírico no rechaza la hipótesis que los establecimientos con un alto porcentaje de clientes repetidores tendrán menor necesidad de los servicios de un intermediario para distribuir su capacidad de alojamiento. La lógica es doble: los clientes que repiten ya disponen de buena información respecto a algunas de las características del hotel en cuestión y, además, los costes para los hoteles de engañar (estafar) son mayores debido la consecuente importante pérdida de negocio futuro.

${ }^{11}$ La muestra incluye información respecto a los canales de distribución (bien mediante un tour operador o agencia de viaje, bien directamente) de más de 300 establecimientos hoteleros, información obtenida mediante una encuesta realizada en el año 2004, preguntando a los gestores de los establecimientos hoteleros en cuestión.

12 En la misma línea, en The Economist (2005) también se muestra como grandes empresas turísticas (cadenas hoteleras y aerolíneas, por ejemplo) venden una gran parte de su capacidad directamente a través de Internet.

${ }^{13}$ Esto implica, por ejemplo, que el análisis realizado por O'Connor y Frew (2002) sobre las perspectivas de la venta on-line de alojamiento hotelero es probable que sea parcial a causa de su enfoque en las grandes cadenas hoteleras. 


\section{LAS TIC Y LA PRODUCTIVIDAD DE LOS INTERMEDIARIOS}

La literatura (académica o no) ha puesto mucho énfasis en el impacto de internet y las TIC en el funcionamiento de los mercados. Así, internet contribuiría a que los distintos mercados funcionaran de una forma mucho más eficiente; en algunos casos internet debería implicar mercados casi de competencia perfecta («frictionless comerce»). Sin embargo, si el interés yace en el impacto de internet en la cadena de valor del sector turístico (o cualquier otro) y en la permanencia y la forma de intermediación, el análisis debe prestar atención no solamente al impacto de internet en el funcionamiento de los mercados, si no también al impacto de internet (y las TIC en general) en el funcionamiento de las empresas u organizaciones en general, entre ellas los intermediarios.

Existe ya una cierta literatura académica que analiza el impacto de las TIC en la productividad empresarial (véase, por ejemplo, Aral et al., 2006), y el impacto de las TIC en el funcionamiento de los distintos intermediarios turísticos viene de lejos, tal y como hemos mencionado más arriba. Las primeras aplicaciones de las TIC (por ejemplo, los 'Global Distribution Systems' y los 'Central Reservation Systems') previa a la aparición de internet fueron aplicaciones que afectaron directamente a los intermediarios, facilitando en gran medida su funcionamiento e incrementando su productividad. Además, desarrollos y aplicaciones posteriores de las TIC y de internet han tenido un gran impacto en el funcionamiento de los intermediarios (y su productividad y eficiencia) en dos líneas principales.

En primer lugar, la adopción de internet y otras TIC ha permitido reducir costes operativos de los intermediarios de manera importante. Un ejemplo entre varios sería el departamento de booking (reservas) de los tour operadores. La adopción de las TIC ha permitido reducir de manera muy importante el personal destinado a los departamentos de booking debido a que, hoy en día, una alta proporción del producto de los tour operadores se vende ya mediante interfície web con las agencias de viajes.

En segundo lugar, y tan o más importante que el impacto en la reducción de costes, las TIC e internet han permitido a los tour operadores llevar a cabo una mayor personalización, una mayor adaptación del producto o paquete turístico) a la demanda, a las preferencias de los consumidores, los turistas. Esto es lo que se denomina dynamic packaging (paquete dinámico) en el que el propio turista se va construyendo el paquete turístico a su medida. Antes, tal y como hemos explicado más arriba, los tour operadores ofrecían paquetes muy estandarizados, por ejemplo en lo que respecta a fechas (paquetes de 7 o 14 días). Ahora, en cambio, el cliente puede elegir las fechas y el 
número de días del viaje, y también los componentes del paquete que le interesan, no incluyendo otros que consigue de forma alternativa (por ejemplo, el vuelo) ${ }^{14}$.

Así pues, es indudable que internet y las otras TIC han tenido un importante y positivo impacto en el servicio y el funcionamiento de los intermediarios turísticos, permitiendo una mayor creación de valor de éstos en el sector turístico, contribuyendo a posibilitar su pervivencia en el futuro.

\section{Comentarios Finales}

El análisis económico y, más concretamente, la teoría de los costes de transacción nos ha permitido analizar y comprender el papel que tradicionalmente han desempeñado los intermediarios (tour operadores) en la cadena de valor turística. A continuación, y como objetivo central del artículo, hemos analizado el impacto que han tenido y previsiblemente tendrán en la cadena de valor del producto turístico el desarrollo de las distintas tecnologías de la información y comunicaciones, y muy especialmente internet.

Tradicionalmente, antes de internet, la función de los tour operadores ha consistido en: (i) coordinar el ajuste de las distintas partes del paquete turístico, (ii) ofrecer información al turista respecto a las características de este paquete, y (iii) aportar reputación para informar de forma creíble respecto a la calidad de los servicios que integran el paquete turístico. Esta labor los tour operadores la llevaban a cabo en gran medida (al menos en el turismo vacacional) mediante la venta/distribución de paquetes estandarizados (que incluían alojamiento, transporte, etc.) y que llegaban al turista a través de las agencias de viaje.

Claramente, internet y las otras TIC han supuesto cambios importantes en la organización de la cadena de valor del producto turístico. Internet facilita la coordinación por parte del propio turista de las distintas partes del producto turístico, así como la búsqueda on-line de información respecto a las características del producto (precios, localización). Esto ha tenido el efecto de reducir la presencia de los intermediarios en

14 Un ejemplo de esta personalización llevada al extremo es el hecho que antes los tour operadores solamente ofrecían paquetes completos, mientras que ahora también ofrecen productos con solamente un único componente (por ejemplo, «sólo hotel»). Esto es así a pesar que en tales casos el margen es muy pequeño, pero se trata de un mecanismo de fidelización del cliente, puesto que es un producto que el cliente demanda. 
la cadena de valor y, de hecho, los datos muestran como el sector turístico se encuentra en un proceso de desintermediación por el que el turista accede directamente a los vendedores finales (hoteles, aerolíneas, etc.).

Aún así, y a pesar de las posibilidades que internet ofrece en el sector turístico, esto no debería llevar a la completa desaparición de los intermediarios predicha por algunos. Incluso en la era de internet los intermediarios tienen razón de ser y pueden aportar valor al turista. Este valor de la intermediación procede de un doble camino. Por un lado, los intermediarios juegan un papel clave en la provisión de información creíble y veraz mediante su reputación. Con internet, la incertidumbre respecto a la calidad a la que se enfrentan los consumidores no desaparece y esto, por lo tanto, posibilita que los tour operadores inviertan en reputación frente a los consumidores para distribuir el producto turístico con garantías de calidad. Por otro lado, es importante reseñar que internet y las TIC ofrecen también a los intermediarios nuevas posibilidades de incrementar su eficiencia y la calidad y variedad de sus servicios (por ejemplo, el 'dynamic packaging') lo cuál debería permitir a los intermediarios coexistir en la cadena de valor junto con los intercambios directos entre turistas y vendedores finales.

Así, aunque a buen seguro la intermediación turística se verá sujeta a importantes cambios (desaparición de alguno de los más importantes tour-operadores, fusiones, intermediarios on-line, etc.), la pervivencia de la intermediación en la cadena de valor de la actividad turística no debería estar en peligro. Es evidente que, sin embargo, nuestro análisis no indica nada respecto al tipo de intermediarios que existirán con internet. The Economist (2005), por ejemplo, proporciona evidencias sobre el papel cada vez mayor de internet en la reserva de servicios de viaje en los EE.UU. Sin embargo, una parte muy importante de las reservas se hace a través de agencias de viajes o tour operadores on-line, lo cual encaja perfectamente dentro de nuestro análisis. Finalmente, reseñar también la aparición reciente de nuevos intermediarios on-line (por ejemplo, Trip Advisor) dentro de lo que es la web 2.0 en la que es clave la interacción directa entre los consumidores. Todo esto sugiere que en el futuro la cadena de valor del producto turístico seguirá experimentando nuevos cambios de gran importancia, aportando nuevas posibilidades en lo que respecta a sus formas organizativas.

\section{REFERENCIAS BIBLIOGRÁFICAS}

Aguilo, E., Alegre, J., and SARd, M., (2003): «Examining the Market Structure of the German and UK Tour Operating Industries through an Analysis of Package Holiday Prices»; Tourism Economics, September v. 9, iss. 3, pp. 255-78. 
Akerlof, G. (1970): «The Market for 'Lemons': Quality Uncertainty and the Market Mechanism»; Quarterly Journal of Economics, 84(3), pp. 488-500.

Aral, S., Brynjolfsson, E. and V. Alstyne, Marshall W., 2006, «Information, Technology and Information Worker Productivity: Task Level Evidence». Available at SSRN: http://ssrn. com/abstract $=942310$.

BAUm, T. and BudAmBI, R. (1994): «A Ricardian analysis of the fully inclusive tour industry», Service Industries Journal, vol. 14, no1, pp 85-93.

Biglaiser, G. (1993): «Middlemen as Experts», Rand Journal of Economics, 1993, 24 (2), páginas 212-23.

Buhalis, D., and Licata, C., (2002): «The future of eTourism intermediaries»; Tourism Management, Vol.23(3), pp.207-220.

Calveras, A. and Orfila, F. Intermediaries and Quality Uncertainty: Evidence from the Hotel Industry (March 25, 2007). Available at SSRN: http://ssrn.com/abstract=1009647.

Carlson, J. A. and McAfee, R. P.; «Discrete Equilibrium Price Dispersion»; The Journal of Political Economy 91; 1983; 480-493.

Clerides, S., Nearchou, P and Panos Pashardes (2006): «Intermediaries as Quality Assessors: Tour Operators in the Travel Industry», Internatinal Journal of Industrial Organization.

COASE, R. (1937): «The nature of the firm»; Economica.

Davidow, W.H. and Malone, M.S., (1992), The Virtual Corporation, (Harper Collins Publishers, New York).

Evans, P., and Wurster, T. S. (2000). Blown to Bits - How the New Economics of Information Transforms Strategy. Boston, MA: Harvard Business School.

Gibbons, R. (2000): «Why Organizations Are Such a Mess (and What an Economist Might do About It)»; chapter 1 of Organizational Economics (a text in progress).

Holmstrom, B., (1979): Moral Hazard and Observability.« Bell Journal of Economics 10:7491.

Huston, J. H.; Spencer, R. W. (2002): «Quality, Uncertainty and the Internet: The Market for Cyber Lemons»; American Economist, Spring 2002, v. 46, iss. 1, pp. 50-60.

KREPS, D. and R. WiLSON (1982), Reputation and Imperfect Information, Journal of Economic Theory, 50: 253-79.

MelniK, M. I.; Alm, J. (2002): «Does a Seller‘s Ecommerce Reputation Matter? Evidence from eBay Auctions»; Journal of Industrial Economics, September, v. 50, iss. 3, pp. 337-49

- (2005): «Seller Reputation, Information Signals, and Prices for Heterogeneous Coins on eBay»; Southern Economic Journal, October, v. 72, iss. 2, pp. 305-28.

Milgrom, P. and Roberts J. (1992): Economics, organization and management. Prentice Hall, Inc. 
Nelson, P. (1970): «Information and consumer behaviour»; Journal of Political Economy 78: 311-329.

- (1974): «Advertising as information»; Journal of Political Economy 82, iss.4.

O’Connor, P. and Frew, A. J., (2002): «The Future of Hotel Electronic Distribution: Expert and Industry Perspectives»; Cornell Hotel and Restaurant Administration Quarterly 43: 33-45.

Renshaw, MB (1994): «Consequences of integration in UK tour operating»; Tourism Management 15 (4).

Sheldon, P. J. (1986): «The Tour Operator Industry: An Analysis», Annals of Tourism Research.

The ECONOMIST (2005), «Flying from the computer», September 29th.

Tirole, J. (1999), «Incomplete Contracts: Where do we Stand?,» Econometrica 67, 741-81.

Whinston A., Choi S. and Stahl D. (1997): The economics of electronic commerce; Macmillian Technical Publishing.

Williamson, O. (1975): Markets and hierarchies, analysis and antitrust implications: A study in the economics of internal organization. Free Press (New York).

Wolinski, A. (1983): «Prices as signals of product quality», Review of Economic Studies 50.

World Tourism Organisation, 2006, Tourism highlights, 2006 edition. 